I am not competent to pass judgment on this sweeping prophecy, but there is no doubt that much interesting and convincing evidence and arguments tending to support it are presented. And on the way, a host of intriguing ideas and speculations, the majority of them not followed up alas, are thrown up.

Here, only a few examples can be given. Few philosophers of mind, not to mention neuropsychologists and experimental psychologists, have yet taken on board the insight provided by the fact that the same cognitive computer programme can be run successfully on different computers with quite different basic components and architecture. In other words, the specifically mental part of the system is in an important sense quite independent of the physical part (which of course is not to say that it can operate without a physical part). How this comes about, the notions of "virtual machine", and of levels of representation of the activity, connected by translators, compilers and interpreters, are of significance here. Consider also the implications for the doctrine of reductionism in the philosophy of science. If biological processes are computational processes running on a physicochemical system, then it is not necessarily the case that the nature of the physical world determines them.

Another important notion for the psychology and philosophy of mind is that of systems and mechanisms which have the property of "mutual recursion", that is, the relationships between any parts of the system are not confined to being disjoint, or part-whole or overlapping, but A can be a part of B which is at the same time a part of $A$, infinite regresses being avoided by stopping conditions. A characteristically fascinating aside of Sloman's here is the suggestion that neglect of this aspect of the mind might have profound implications for educational practice, in that cumulative educational programmes may be misguided: teachers should not be surprised at pupils getting things wrong when they are in the midst of learning a collection of mutually recursive concepts.

An example of how tantalising the author can be is in a chapter which contains a most original and searching analysis of the relationships between inferences of the analogical type, such as those using diagrams, in contrast to the more usual "logical" types. In the middle he makes the very interesting suggestion, without following it up at all, that the essence of our notion of causality may be the following: we talk of causes when we believe there is a representation of the process (in the analogical or other form) which enables the effect to be inferred in the broader sense of "logic" he has been developing.

In the hope that the above has given some sense of the flavour of the book,
I shall now give a very brief summary of its contents. The first section deals with the aims of science and its relation to philosophy, putting the emphasis on the study of possibilities rather than the search for laws, and on conceptual analysis; it incidentally argues for a closer community of aims among philosophy, science and technology. The next section first attempts (in a very ambitious way) to sketch the structure of a mechanism of general intelligence. Three successive chapters then discuss intuition and analogical reasoning, learning about numbers and its bearing on the many and very diverse philosophical views on the nature of number, and perception as a computational process. It seems clear that the latter topic (in which Sloman's own research has been mainly concentrated) has been the one that has stimulated his thinking most. The final chapter discusses a large set of classical problems of philosophy in the light of our present understanding of artificial intelligence. These include universals, free will and determinism, mathematical knowledge, and aesthetic experience.

The main criticism one might make of the book, as has probably already occurred to the reader of this review,

\section{Mathematical approach to vectors}

Vectors in Three-Dimensional Space. By J. S. R. Chisholm. Pp.293. (Cambridge University Press: Cambridge and London, 1978.) Hardback £15; paperback $£ 4.95$.

FEw can appreciate a Beethoven sonata by merely reading the score. It needs to be played by a gifted interpreter. Ease and familiarity with mathematical ideas come quickly to only a few. Many of us need to develop mathematical skills, but such skills are precariously based if we have no appreciation of the underlying mathematical logic. The interpreter is needed here too and J. S. R. Chisholm has demonstrated yet again that he has this gift. His book with Morris Mathematical Methods in Physics - now sadly out of print-was a model of its kind: clear and readable but paying attention always to the rigorous basis of the techniques. This new volume is aimed more directly at the mathematics undergraduate but it should be one of the 'lecturer's textbooks' for anyone teaching vectors to scientists and engineers.

Too often a mathematician's approach to vectors begins with an assertion of the general algebra of vector spaces, leaving the student to find analogies, if he can, with the is that it tries to cover too much, with the result that it spreads itself too thin over the large majority of the issues it raises, and gives the impression of making sweeping judgments without sufficient backing. Sloman also has a maddening habit of producing frequently lists (sometimes as long as fifty items) of questions for the reader, most of which are indeed interesting and pertinent ones, but of few of them does he say anything more. In addition, nearly every one of them would probably require at least a day's solid thinking on the reader's part to get any benefit from. Also, future editions of the book (which I am confident will be called for) would be improved by correction of the inordinate number of misspellings, removal of howlers like attributing the explanation of the equivalence of mass and energy to the theory of general relativity, or stating that superconductivity was explained before it was observed, and also improving the legibility of the legends of the diagrams.

Bernard Meltzer

Bernard Meltzer is Professor of Computational Logic in the University of Edinburgh, UK.

world of three dimensional vector quantities in which he lives. This book maintains a healthy link with intuition. The axioms of vector space algebra are introduced from the start, but the three-dimensional geometrical interpretation of them takes up much of the text, with line-drawings to illustrate many of the arguments.

The relationship between vectors and vector-quantities is clearly explained. After establishing the axioms and discussing the products of vectors, there is a chapter in which geometrical examples are used to illustrate the relationship of vectors to matrices, tensors, the rotation group and general linear transformations. Vector calculus is introduced with a discussion of the geometry of curves and surfaces, going on to the the differential geometry of curves, surface integrals and volume integrals.

The final chapter on vector analysis introduces the div grad and curl of scalar and vector fields in three dimensions, with Stokes's Theorem (a more rigorous proof is given as an appendix), the Laplace operator and related theorems.

Most subsections of each chapter have worked examples and up to seven or eight problems. Short answers to many of the problems are given at the back of the book.

David J. Miller

David J. Miller is Lecturer in Physics at University College, London, UK. 